\title{
A novel sling technique for microvascular decompression of a rare anomalous vertebral artery causing cervical radiculopathy
}

\author{
Adesh Tandon, M.D., ${ }^{1}$ Sid Chandela, M.D., ${ }^{2}$ David Langer, M.D., ${ }^{3}$ \\ and Chandranath Sen, M.D. ${ }^{4}$ \\ ${ }^{1}$ Department of Neurosurgery, Albert Einstein College of Medicine, Bronx, New York; ${ }^{2}$ Virginia \\ Neurosurgeons P.C., Arlington, Virginia; ${ }^{3}$ Department of Neurosurgery, Hofstra University School of \\ Medicine, Manhasset, New York; and ${ }^{4}$ Department of Neurosurgery, New York University School of Medicine, \\ New York, New York
}

Cervical radiculopathy secondary to compression from congenital anomalous vertebral arteries (VAs) is a known entity. Patients present with a variety of symptoms ranging from upper-extremity numbness to true occipital neuralgia. Treatment options for extracranial tortuous VAs include conservative management or some form of surgical microvascular decompression (MVD). The authors report on a patient with a congenital anomalous VA loop causing cervical nerve root compression. Successful MVD was conducted with relief of the patient's symptoms. A novel sling technique was used for mobilization of the VA. To the authors' knowledge, this is the first MVD described utilizing this technique.

(http://thejns.org/doi/abs/10.3171/2013.6.FOCUS1339)

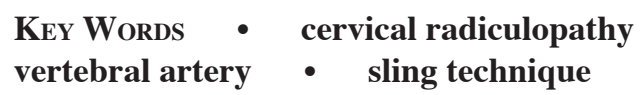

- microvascular decompression •
Imaging Studies. Magnetic resonance imaging of the cervical spine revealed mild degenerative spondylosis with evidence of a flow void in the right $\mathrm{C} 4-5$ neural foramen. Further radiographic studies, including CT angiography and MRI, demonstrated bilateral tortuous VAs. There was additional evidence of an anomalous VA loop within the right C4-5 neural foramen, likely compressing the exiting nerve root at this level (Fig. 1A and B). Given the failure of conservative therapy, the decision was made to recommend surgical intervention; more specifically, the recommendation for an anterior-lateral approach was made, accompanied by an MVD.

Operation. The patient was taken to the operating room for a right-sided anterolateral cervical approach for MVD at the C4-5 neural foramen. A right-sided, vertical, anterior cervical incision was planned along the medial border of the sternocleidomastoid muscle. Once the middle cervical fascia was bluntly dissected, the carotid sheath was reflected posteriorly and the vertebral transverse foramina were easily palpated. Utilizing intraoperative fluoroscopy, the noted levels were confirmed. The C-4 and C-5 vertebral bodies were identified and the corresponding transverse foramina were localized. The VA was identified as well as the exiting nerve root at that level. A high-speed diamond drill was used to skeletonize the VA from the transverse foramina (Fig. 1C). The VA was

\footnotetext{
Abbreviations used in this paper: $\mathrm{MVD}=$ microvascular decompression; VA = vertebral artery.
} 

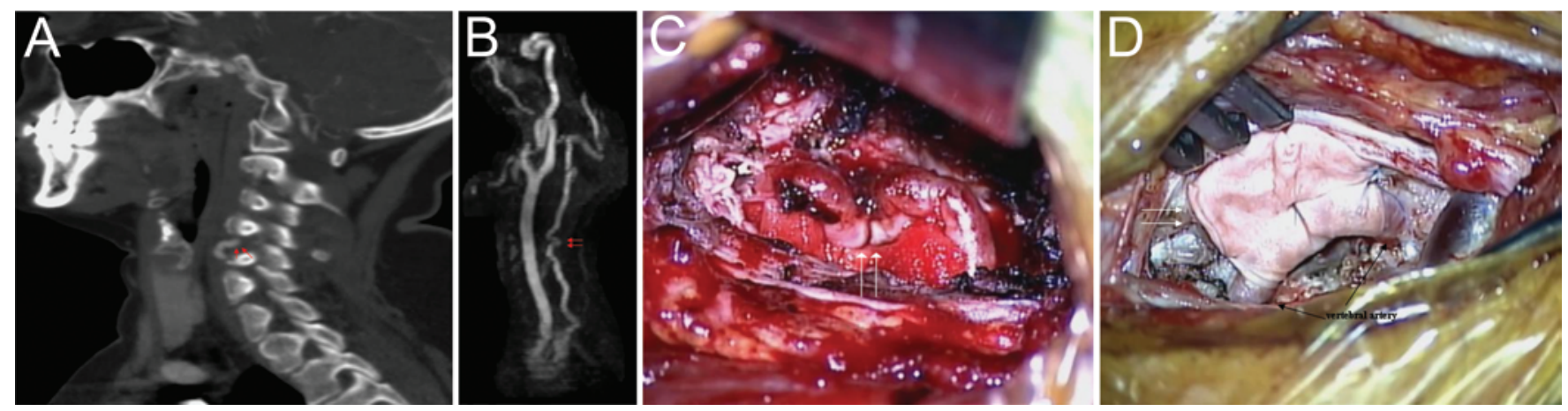

FIG. 1. A: Sagittal CT angiography of the patient's cervical spine illustrating an anomalous VA loop entering the C4-5 intervertebral foramen (arrows). B: Magnetic resonance angiography of the neck reveals an anomalous VA loop (arrows). C: Intraoperative view of the anomalous loop of the VA at the level of C4-5 (arrows). D: Intraoperative view of the acellular human dermis sling (double arrows) created around the VA, gently pulling it away from the intervertebral foramen.

isolated and transposed away from the exiting nerve root. Next, a sling was created utilizing an acellular human dermis allograft. This sling was gently wrapped around the VA and interrupted sutures were used to sew the sling to the adjacent paraspinal muscle (Fig. 1D). The VA was easily mobilized and secured. Following the decompression, an intraoperative indocyanine green angiography study confirmed brisk flow through the VA.

Postoperative Course. The patient's symptoms resolved after surgery. On a 1-month follow-up visit the patient stated that her neck and right arm pain had resolved completely. No apparent deficits were noted upon physical examination. On 1-year follow-up the patient noted similar resolution of symptoms.

\section{Discussion}

Cervical radiculopathy is defined as a dysfunction of a nerve root of the cervical spine. Most commonly, the cause of cervical radiculopathy is cervical disk herniations and/or osteophyte formation., ${ }^{1,7-9}$ Other causes of cervical radiculopathy include intervertebral neurofibromas, metastatic tumors, and/or trauma. ${ }^{7,8}$ A common radiographic finding associated with these lesions is enlargement of the associated neural foramen., , $^{1,5,7-9}$

Vertebral artery tortuosity associated with cervical radiculopathy is a rare entity. An ectatic VA loop can impinge upon the neural foramen and directly compress the exiting nerve root. ${ }^{1-9}$ Thus, the idea of direct vascular decompression appears to be a viable option for treatment of this condition. Although the literature on this topic is sparse, a few case reports illustrate successful treatment through microvascular decompressive surgeries. ${ }^{1-9}$

Sharma et al. reported on a 75-year-old woman with a multiyear history of occipital neuralgia. ${ }^{9}$ On radiographic imaging it was noted that an ectatic VA was compressing the exiting nerve root at $\mathrm{C} 1-2$. A posterior MVD was conducted, with the placement of an oxidized cotton plug to separate the exiting root from the vessel. The patient's neuralgia resolved postoperatively and at the 18-month follow-up evaluation the patient remained asymptomatic. Duthel et al. reported on a 37-year-old man with persistent left shoulder pain and tingling sensations in his hand. ${ }^{2}$ This patient's radiographic studies demonstrated an enlarged intervertebral foramen at C5-6. Further studies of the surrounding vascular structures demonstrated an aberrant loop of the VA impinging upon the C5-6 intervertebral foramen. This patient underwent an anterolateral cervical decompression. The nerve root and VA were dissected away from each other and a Teflon plug was used to maintain separation.

Recently, Korinth and Mull reported on a 68-yearold woman with a 3-year history of right-sided neck and shoulder pain. ${ }^{6}$ All conservative therapy failed to relieve this patient's symptoms. Magnetic resonance angiography confirmed the diagnosis of a VA loop causing neural compression at the $\mathrm{C} 4-5$ intervertebral foramen. This patient also underwent an anterolateral approach for direct MVD. The patient's symptoms resolved postoperatively with no signs of recurrence on follow-up.

We present the case of cervical radiculopathy secondary to direct neural compression from an aberrant loop of the VA. Our patient underwent a successful anterolateral decompression as well, but we describe a novel technique we used to maintain physiological separation. In our case, an acellular human dermis allograft sling was fashioned to maintain separation of the VA from the exiting nerve root. This sling was carefully secured to the surrounding tissue to maintain a constant separation, so that the nerve would not be subjected to the pulsatile flow of the VA. An intraoperative indocyanine green angiography study was conducted to confirm VA patency. The advantage of this technique is that it allows for a more stable maintenance of physiological separation then those techniques previously described (such as a Teflon plug, or oxidized cotton plug).

Our case illustrates the utility of MVD in cases of aberrant VA loops causing neuronal compression. Suspicion should be raised if the cervical radiculopathy presents as atypical, without evidence of symptomatic cervical spondylosis. Furthermore, radiograph studies should be used to confirm the presence of aberrant vascular anatomy. In treating this pathology, our case illustrates that MVD in conjunction with an acellular dermis sling should be considered as a treatment option.

\section{Disclosure}

The authors report no conflict of interest concerning the mate- 


\section{Microvascular decompression of a rare anomalous vertebral artery}

rials or methods used in this study or the findings specified in this paper.

Author contributions to the study and manuscript preparation include the following. Conception and design: all authors. Acquisition of data: all authors. Analysis and interpretation of data: all authors. Drafting the article: all authors. Critically revising the article: all authors. Reviewed submitted version of manuscript: all authors. Approved the final version of the manuscript on behalf of all authors: Tandon.

\section{References}

1. Detwiler PW, Porter RW, Harrington TR, Sonntag VKH, Spetzler RF: Vascular decompression of a vertebral artery loop producing cervical radiculopathy. Case report. J Neurosurg 89:485-488, 1998

2. Duthel R, Tudor C, Motuo-Fotso MJ, Brunon J: Cervical root compression by a loop of the vertebral artery: case report. Neurosurgery 35:140-142, 1994

3. Fink JR, Leung JY, Creutzfeldt CJ: Vertebral artery loop formation causing severe cervical nerve root compression. Neurology 75:192, 2010

4. Horgan MA, Hsu FP, Frank EH: Cervical radiculopathy secondary to a tortuous vertebral artery. Case illustration. J Neurosurg 89:489, 1998
5. Kim HS, Lee JH, Cheh G, Lee SH: Cervical radiculopathy caused by vertebral artery loop formation: a case report and review of the literature. J Korean Neurosurg Soc 48:465468,2010

6. Korinth MC, Mull M: Vertebral artery loop causing cervical radiculopathy. Surg Neurol 67:172-173, 2007

7. Paksoy Y, Levendoglu FD, Ogün CO, Ustün ME, Ogün TC: Vertebral artery loop formation: a frequent cause of cervicobrachial pain. Spine (Phila Pa 1976) 28:1183-1188, 2003

8. Sakaida H, Okada M, Yamamoto A: Vascular reconstruction of a vertebral artery loop causing cervical radiculopathy and vertebrobasilar insufficiency. Case report. J Neurosurg 94 (1 Suppl):145-149, 2001

9. Sharma RR, Parekh HC, Prabhu S, Gurusinghe NT, Bertolis $\mathrm{G}$ : Compression of the $\mathrm{C}-2$ root by a rare anomalous ectatic vertebral artery. Case report. J Neurosurg 78:669-672, 1993

Manuscript submitted January 30, 2013.

Accepted June 11, 2013.

Please include this information when citing this paper: DOI: 10.3171/2013.6.FOCUS1339.

Address correspondence to: Adesh Tandon, M.D., Department of Neurosurgery, Albert Einstein College of Medicine, 3316 Rochambeau Ave., Bronx, NY 10467. email: atandon26@gmail.com. 